\title{
Stent Thrombosis Coronary ARC Grade Clinical Classification
}

National Cancer Institute

\section{Source}

National Cancer Institute. Stent Thrombosis Coronary ARC Grade Clinical Classification.

NCI Thesaurus. Code C119107.

A standardized grading system developed by the Academic Research Consortium (ARC) to describe coronary stent thrombosis based on the evidence (angiographic or pathological confirmation of thrombus, or unexplained sudden death within 30 days) of the event. This grading system includes 3 classifications: definite, probable and possible stent thrombosis. 